\title{
Parametric excitation of plasma oscillations in a Josephson tunnel junction
}

\author{
Bak, Christen Kjeldahl; Kofoed, Bent; Pedersen, Niels Falsig; Særmark, Knud
}

Published in:

Journal of Applied Physics

Link to article, DOI:

$10.1063 / 1.321609$

Publication date:

1975

Document Version

Publisher's PDF, also known as Version of record

Link back to DTU Orbit

Citation (APA):

Bak, C. K., Kofoed, B., Pedersen, N. F., \& Særmark, K. (1975). Parametric excitation of plasma oscillations in a Josephson tunnel junction. Journal of Applied Physics, 46(2), 886-889. https://doi.org/10.1063/1.321609

\section{General rights}

Copyright and moral rights for the publications made accessible in the public portal are retained by the authors and/or other copyright owners and it is a condition of accessing publications that users recognise and abide by the legal requirements associated with these rights.

- Users may download and print one copy of any publication from the public portal for the purpose of private study or research.

- You may not further distribute the material or use it for any profit-making activity or commercial gain

- You may freely distribute the URL identifying the publication in the public portal

If you believe that this document breaches copyright please contact us providing details, and we will remove access to the work immediately and investigate your claim 


\title{
Parametric excitation of plasma oscillations in a Josephson tunnel junction
}

\author{
C. K. Bak, B. Kofoed, N. F. Pedersen, and K. Saermark \\ Physics Laboratory I, Technical University of De'nmark, DK-2800 Lyngby, Denmark \\ (Received 16 September 1974)
}

\begin{abstract}
Experimental evidence for subharmonic parametric excitation of plasma oscillations in Josephson tunnel junctions is presented. The experiments described are performed by measuring the microwave power necessary to switch a Josephson-tunnel junction biased in the zero-voltage state to a finite-voltage state.
\end{abstract}

\section{INTRODUCTION}

The use of a Josephson junction as a parametric amplifier has been investigated previously, theoretical$1 y^{1-3}$ as well as experimentally. ${ }^{2,4,5}$ In Refs. 1, 2, 4, and 5 the Josephson frequency $\omega_{0}=2 e V_{0} / \hbar$ is used as the pump frequency and an external cavity is used as the input circuit. Here $e$ is the electron charge, $2 \pi \hbar$ is Planck's constant, and $V_{0}$ is the dc voltage across the junction. In Ref. 3 the possibility of using the plasma frequency in a Josephson junction as the input circuit and an external signal as the pump has been investigated theoretically, and it was suggested that parametric excitation of the plasma oscillations should be observable in a Josephson thin-film junction but less likely in point contacts. One advantage of using the plasma frequency as the input resonance frequency is the possibility of a rather easily performed tuning by varying the dc Josephson current. In the present paper experimental evidence for parametric excitation of plasma oscillations in the thin-film Josephson junctions is presented. The paper is organized along the following lines: In Sec. II the experimental details are presented. In Sec. III the experimental results are presented and discussed. Finally, Sec. IV contains the conclusion.

\section{EXPERIMENTAL DETAILS}

\section{A. Experimental setup}

The samples were $\mathrm{Sn}-\mathrm{Sn}_{x} \mathrm{O}_{y}-\mathrm{Sn}$ and $\mathrm{Nb}-\mathrm{Nb}_{x} \mathrm{O}_{y}-\mathrm{Pb}$ cross-type thin-film tunnel junctions. The $\mathrm{Sn}_{-}-\mathrm{Sn}_{x} \mathrm{O}_{y}-\mathrm{Sn}$ junctions were prepared by evaporation in vacuum $\left(10^{-6}\right.$ Torr). The barrier was produced by letting the first deposited film oxidize for about $20 \mathrm{~h}$ in an atmosphere of pure oxygen at $50^{\circ} \mathrm{C}$. The effective tunneling area was $1.5 \times 10^{-7} \mathrm{~m}^{2}$ and the maximum dc Josephson current about $6 \mathrm{~mA}$ at $1.6{ }^{\circ} \mathrm{K}$. This current was approximately $80 \%$ of the theoretical maximum current. The plasma frequency of the junctions was less than $14 \mathrm{GHz}$ and the lowest geometrical resonance was measured to be 20 $\mathrm{GHz}$. The dimensions of the junctions were $0.3 \times 0.5$ $\mathrm{mm}^{2}$. The $\mathrm{Nb}-\mathrm{Nb}_{x} \mathrm{O}_{y}-\mathrm{Pb}$ junctions were prepared by plasma oxidation of the $\mathrm{Nb}$ film as described in Ref. 6.

The experiments were performed at frequencies of $8.5-11 \mathrm{GHz}$ and at frequencies of $2-4 \mathrm{GHz}$ using a Gunn diode (Philips PM 7015x) and a S-band generator (Sivers Lab PM 7008 S), respectively. The equipment consisted, further, of an isolator, a directional coupler for monitoring the power output, and a calibrated attenuator. The measurements were done in a shielded room.
The double-glass Dewar system was surrounded by a two-layered mu-metal can to screen out the earth's magnetic field, and a pair of Helmholtz's coils were placed in the liquid-nitrogen Dewar providing for magnetic fields up to $50 \mathrm{Oe}$, when desired.

The microwaves were introduced into the cryostat through a low-temperature coaxial cable and coupled to the junction by a loop surrounding the junction, with the junction placed in the middle of the loop.

\section{B. Experimental procedure}

According to the simple theory $I_{\mathrm{dc}}^{\max }$ is proportional to $J_{0}\left(2 e V_{\mathrm{rf}} / \hbar \omega\right)$, where $J_{0}$ is the Bessel function of order zero. Measurement of $I_{d c}^{\max }$ as function of the square root of the applied microwave power should give this dependence. ${ }^{7}$ In general a remarkable variety of singularities and hysteresis effects have been observed for example as reported by Dahm et al. ${ }^{8}$

In tracing the $I-V$ characteristic on an oscilloscope certain intervals of the dc Josephson current showed instabilities with applied microwaves. Biasing the junction in these intervals was impossible. In order to make the measurements in a reproducible way the junction was biased with a fixed dc current, $I_{d c}$, at zero voltage without any microwaves present. The microwave power was then gradually increased until the junction switched to a voltage different from zero. An example of measurements performed in this way is shown in Fig. 1. Here the $\mathrm{dB}$ reading of the calibrated attenuator when the junction switched is plotted as function of the dc bias current, $I_{\mathrm{dc}}$, the microwave frequency being kept con-

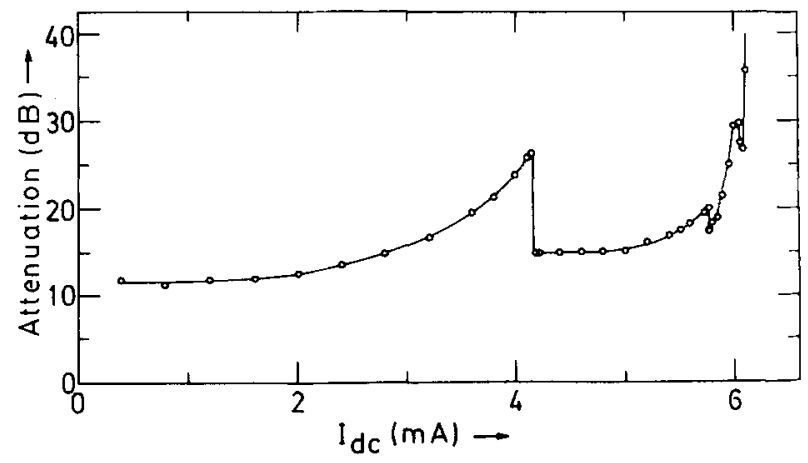

FIG. 1. Inserted attenuation in $\mathrm{dB}$ of the applied microwave power necessary for switching the junction as a function of the dc bias current $I_{\mathrm{dc}}$ at zero voltage for a $\mathrm{Sn}-\mathrm{Sn}_{x} \mathrm{O}_{y}-\mathrm{Sn}$ tunnel junction. 

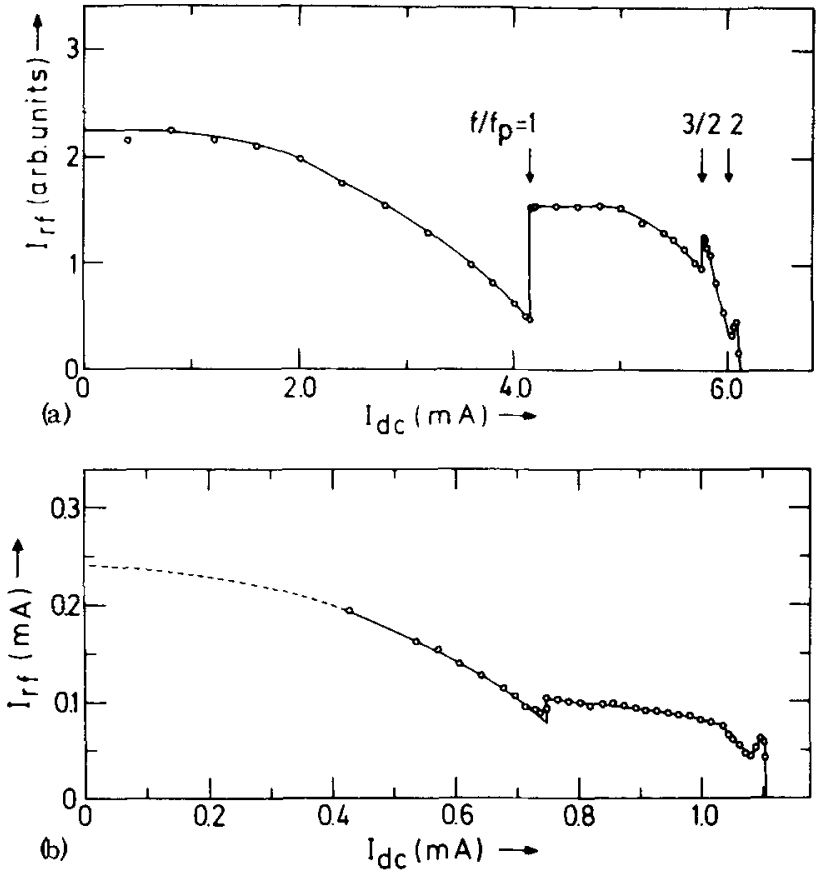

FIG. 2. (a) The square root of the microwave power, i.e., the rf current in arbitrary units necessary for switching the junction as function of the dc bias current $I_{\mathrm{dc}}$ at zero voltage for the same junction as in Fig. 1. (b) Similar curve as in (a), but measured on a Josephson junction analog $\left(I_{\max }=1.1 \mathrm{~mA}, R_{0}=550 \Omega\right.$, $C=100 \mathrm{nF}, f=11.2 \mathrm{kHz}$.

stant during the measurements. Since it is the $\mathrm{dB}$ reading of the inserted attenuator which is plotted, a maximum point in the curve in Fig. 1 represents a point where the junction demands minimal microwave power for switching to a voltage state. The values of the dc Josephson current, $I_{\mathrm{dc}}^{R}$, at the maximum points are used in the analysis. In order to compare the measurements to similar measurements performed on a Josephsonjunction analog ${ }^{9}$ the square root of the applied microwave power as function of the dc Josephson current has also been plotted. An example is shown in Fig. 2(a). In the following we shall call a curve of this type for a "switching curve". Recently Balkashin et al. ${ }^{10}$ have reported on a similar curve measured in a different way by keeping the microwave power constant and increasing the dc current until the junction switched.

Curves like the ones which have been shown in Figs. 1 and 2(a) have been measured for a number of microwave frequencies in the intervals $2-4$ and $8.5-11 \mathrm{GHz}$.

Measurements as described above have been performed on several thin-film Josephson junctions of the type $\mathrm{Sn}-\mathrm{Sn}_{x} \mathrm{O}_{y}-\mathrm{Sn}$ and $\mathrm{Nb}-\mathrm{Nb}_{x} \mathrm{O}_{y}-\mathrm{Pb}$. All the junctions behaved in the same manner in full agreement with the discussion in Sec. III.

\section{DISCUSSION OF EXPERIMENTAL RESULTS}

Central to the discussion is the Josephson plasma frequency. ${ }^{8}$ it may be written in the form

$$
\omega_{b}^{2}=\frac{2 e_{\mathrm{dc}}^{\max }}{\hbar C}\left[1-\left(\frac{I_{\mathrm{dc}}}{I_{\mathrm{dc}}^{\max }}\right)^{2}\right]^{1 / 2}=\omega_{b, \max }^{2} \cos \phi_{0}
$$

where $I_{\mathrm{dc}}^{\max }$ is the maximum dc Josephson current (supercurrent), $C$ is the capacitance, and $I_{\mathrm{dc}}$ is the dc bias current. In the junction experiments we assume the applied microwaves to be a current source. When the applied microwave frequency is equal to the plasma frequency $\omega \simeq \omega_{p}$, the junction $r f$ voltage is enhanced, and the junction will require comparatively lower microwave power for switching than for $\omega \neq \omega_{p}$. Hence, minima in the switching curve may be expected when the applied frequency is equal to the plasma frequency and, possibly, to harmonics and subharmonics of $\omega_{p}$.

Figure 2(a) shows a typical experimental switching curve obtained in the manner described in Sec. II. The curve shows the square root of the rf power as a function of the dc bias current, $I_{\mathrm{dc}}$. The applied frequency is $10.897 \mathrm{GHz}$ and the junction has $I_{\mathrm{dc}}^{\max }=6.11 \mathrm{~mA}$ and a normal state resistance $R=0.1 \Omega$. Three characteris tic structures are observed in Fig. 2(a), and labelled $\omega / \omega_{0}=2, \frac{3}{2}$, and 1 , respectively. This labelling will be described in detail below. Figure $2(\mathrm{~b})$ is a similar switching curve obtained for a Josephson junction ana$\log ^{9}$; again the analog is biased in the zero-voltage mode and an rf signal is applied until it switches to a voltage state. The analog parameters are given in the caption for Fig. 2(b). A careful investigation of the analog voltage waveform on an oscilloscope shows that the observed structures are related to a large-signal plasma resonance, subject to the condition $\omega=\omega_{p}$, and a parametric subharmonic excitation of the plasma resonance, ${ }^{3}$ subject to the condition $\omega_{p}=\frac{1}{2} \omega$. In addition the analog experiments show the usual harmonic resonances, subject to the condition $n \omega=\omega_{p}$.

In order to unambiguously identify the resonances in the real junctions two methods were employed. One method was to obtain switching curves for a wide frequency range covering both harmonic and subharmonic generation and then determine the indexing that would

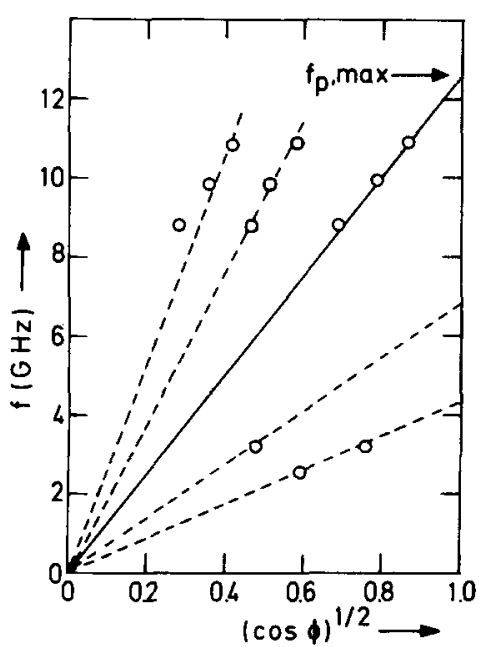

FIG. 3. The points in the diagram are the applied microwave frequency as a function of $\left(\cos \phi_{0}\right)^{1 / 2}$ which is calculated from Eq. (2) using the dc current $I_{\mathrm{dc}}^{R}$ corresponding to the pronounced structure in the switching curves. The extrapolated plasma frequency is $f_{p, \max }=12.6 \mathrm{GHz}$. Note that $\left(\cos \phi_{0}\right)^{1 / 2}=0.3$ corresponds to an $I_{d c}$ current of $99.5 \%$ of the maximum dc current, $I_{\mathrm{dc}}^{\max }$. 


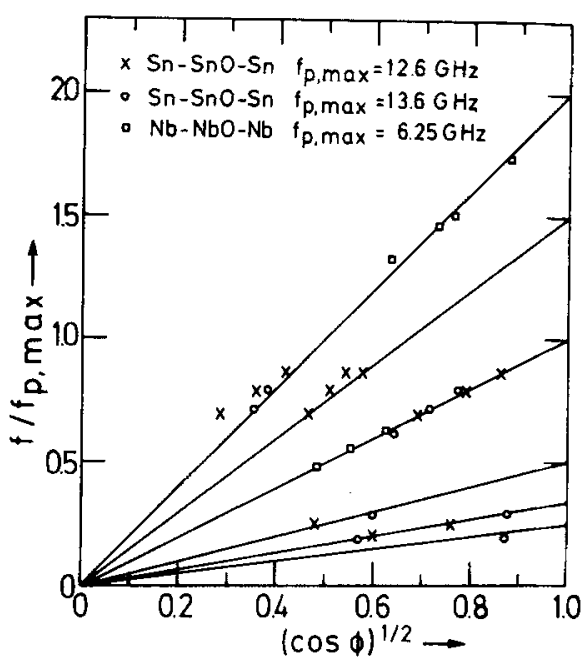

FIG. 4. Experimental points for three different tunnel junctions plotted in the same way as in Fig. 3 , but the microwave frequency is here normalized to the plasma frequency found for each tunnel junction.

fit all of the observed structures. An example of this method is shown in Fig. 3, which shows a plot of three frequencies in the $8.5-11-\mathrm{GHz}$ range and three frequencies in the $2-4-\mathrm{GHz}$ range. For each frequency the dc current, $I_{\mathrm{dc}}^{R}$, corresponding to the pronounced structures (minima in the switching curves) is read and the quantity $\left(\cos \phi_{0}\right)^{1 / 2}$ [proportional to the plasma frequency, Eq. (1)] is calculated as

$$
\left(\cos \phi_{0}\right)^{1 / 2}=\left[1-\left(\frac{I_{d c}^{R}}{I_{\mathrm{dc}}^{\max }}\right)^{2}\right]^{1 / 4}
$$

This plot gives a maximum plasma frequency of 12.6 $\mathrm{GHz}$ and the series $\omega / \omega_{p}=2, \frac{3}{2}, 1, \frac{1}{2}$, and $\frac{1}{3}$ can be identified.

The other method was to determine independently the magnitude of the plasma frequency by measuring the capacitance and inserting in Eq. (1). For the $\mathrm{Nb}-\mathrm{Nb}_{x} \mathrm{O}_{y}-$ $\mathrm{Pb}$ junction $\left(R=0.05 \Omega, I_{\mathrm{dc}}^{\max }=3.3 \mathrm{~mA}\right)$ the capacitance was determined by measuring the capacitance of a highresistance $(R=100 \Omega)$ junction from the same batch directly and extrapolating as done for example in Ref. 11. The results of the two methods agreed within a few percent. In any case an improper indexing would give a factor of 4 in the capacitance - far beyond the experimental uncertainty. Figure 4 shows the results for the three samples that were most extensively studied. The ordinate is the frequency normalized to the plasma frequency. The straight lines correspond to $\omega / \omega_{p}=2, \frac{3}{2}, 1$, $\frac{1}{2}$, and $\frac{1}{3}$, and are not fitted to the data points.

We add that in one case the plasma frequency was changed by applying a magnetic field. The structure in the switching curve shifted in qualitative agreement with the magnetic field dependence of the plasma frequency. ${ }^{12}$

The excitation of the plasma resonance by harmonics of the applied microwave signal is well understood and has been reported earlier. ${ }^{8,12}$ What is new in the present experiments is the excitation of the plasma resonance by means of subharmonic generation, which has been theoretically predicted earlier. ${ }^{3}$ The proposed mechanism is a parametric excitation of the plasma resonance when the applied microwaves have a frequency $\omega=2 \omega_{p}$. For $\omega \lesssim 2 \omega_{\text {o }}$ this parametric subharmonic generation does not increase gradually with junction nonlinearity as harmonic generation does, but appears suddenly with a large amplitude when the microwave power level exceeds a certain threshold value depending on the junction losses, whereas for $\omega \gtrsim 2 \omega_{p}$ the amplitude increases continuously from zero. The detailed calculations will not be reproduced here, but may be found in Ref, 3 . The threshold microwave power may be estimated for the present experiments using

$$
\alpha \tan \phi_{0}>2 / Q \text {, }
$$

where $Q=\omega_{p} R C, \sin \phi_{0}=I_{\mathrm{dc}} / I_{\mathrm{dc}}^{\max }$, and $\alpha=2 e V_{\mathrm{rt}} / \hbar \omega$. For the junction of Fig. 2(a) and for $\tan \phi_{0}=1$, one finds $Q$ $\cong 15$ and $\alpha \geqslant 0.1$ which at $10 \mathrm{GHz}$ corresponds to an $\mathrm{rf}$ voltage amplitude of a few microvolts. This low power level is in qualitative agreement with the observation that the measured plasma frequencies of Figs, 3 and 4 are not significantly shifted from the small-signal resonance frequency.

From analog measurements where the waveform of the rf voltage can be observed on an oscilloscope we know that the threshold value for subharmonic generation does not in general coincide with the rf voltage necessary for switching. Thus the theory of Ref, 3 is not directly applicable for a quantitative comparison, particularly with respect to the amplitude of the $\mathrm{rf}$ voltage and the power threshold curve, For this reason we have not tried to determine the magnitude and sign of the $\cos \phi_{0}$ term $^{12,13}$ although some information may be contained in the experimental curves.

A comment should be made as to the appearance of the structure at $\omega / \omega_{p}=\frac{3}{2}$. This structure does not correspond to a higher-order solution of the Mathieu equation [Eq. (3)], but once the threshold curve corresponding to the excitation at $\omega \cong 2 \omega_{p}$ has been reached it is known that $\mathrm{rf}$ voltage contain frequency components at $\frac{1}{2} \omega, \frac{3}{2} \omega, \frac{5}{2} \omega, \ldots$, where the higher harmonics diminish rapidly in amplitude (Ref. 3 contains information about relative magnitudes for analog measurements). An interesting observation is that for a junction very similar to the one in Fig. 2(a) but containing a short (circles in Fig. 4) the $\frac{3}{2}$ harmonics disappeared, whereas the other harmonics were essentially similar to the junction in Fig. 2(a). This behavior may be expected if the $Q$ of the junction is lowered because of the short.

\section{CONCLUSION}

By measuring switching curves for Josephson tunnel junctions, that is, the microwave power necessary to switch the junction to a finite voltage as a function of the dc current in the zero-voltage mode and analyze the position of the pronounced structure in the curves, it has been possible to identify the structure as belonging to subharmonic generation of the plasma frequency. This identification is further supported by similar measurements on a Josephson-junction analog where the waveform of the rf voltage has been observed. Therefore our conclusion is that we have demonstrated experimentally that the plasma resonance may be excited 
by parametric subharmonic generation as predicted in Ref. 3. This motivates attempts at using a zero voltage, i.e., dc current-biased mode of a Josephson junction as the nonlinear element in a parametric amplifier.

\section{ACKNOWLEDGMENT}

The authors wish to express our sincere thanks to Professor T. Stubb, Helsinki University of Technology, for placing the $\mathrm{Nb}$ junctions at our disposal.

${ }^{1}$ P. Russer, Arch. Elek. Übertragung 23, 417 (1969). ${ }^{2}$ A. N. Vystavkin, V.N. Gubankov, L. S. Kuzmin, K. K. Licharev, and V.V. Migulin, Cornell Conference on High Frequency Generation and Amplification, 1971 (unpublished).
${ }^{3}$ N.F. Pedersen, M.R. Samuelsen, and K. Saermark, J. Appl. Phys. 44, 5120 (1973).

${ }^{4} \mathrm{H}$. Zimmer, Appl. Phys. Lett. 10, 193 (1967).

${ }^{5}$ H. Kanter and A.H. Silver, Appl. Phys. Lett. 19, 515 (1971).

${ }^{6}$ R. Graeffe and T. Wiik, J. Appl. Phys. 42, 2146 (1971). ?S. Shapiro, Phys. Rev. Lett. 11, 80 (1963).

${ }^{8}$ A. J. Dahm, A. Denenstein, T. F. Finnegan, D. N. Langenberg, and D.J. Scalapino, Phys. Rev. Lett. 20, 859 (1968). ${ }^{9}$ C. K. Bak and N. F. Pedersen, Appl. Phys. Lett. 22, 149 (1973).

${ }^{10}$ O. P. Balkashin, L. J. Ostrovsky, and J.K. Yanson, Phys. Condensed State 28, 3 (1973).

${ }^{11}$ P. K. Hansma, G.I. Rochlin, and J.N. Sweet, Phys. Rev. B 4, 3003 (1971).

${ }^{12}$ N. F. Pedersen, T.F. Finnegan, D. N. Langenberg, Phys. Rev. B 5, 4151 (1972).

${ }^{13}$ C.M. Falco, W.H. Parker, and S.E.Trullinger, Phys. Rev. Lett. 31, 933 (1973); D.A. Vincent and B.S. Deaver, Jr., Phys. Rev. Lett. 32, 212 (1974). 